\title{
Technology Used for Blind Indoor Navigation System using Li-Fi
}

\author{
Shilpa $B^{1}$, Manisha M Shetty ${ }^{2}$, Abhijith Shenoy ${ }^{3}$, Rahul Biddappa ${ }^{4}$, Dhanyashree ${ }^{5}$ \\ Assistant Professor, Department of ISE, Canara Engineering College, Benjanapadavu, Mangalore, India ${ }^{1}$
}

Student, Department of ISE, Canara Engineering College, Benjanapadavu, Mangalore, India ${ }^{2}$

\begin{abstract}
Navigation in indoor environments is highly challenging for the severely visually impaired, particularly in spaces visited for the first time. Several solutions have been proposed to deal with this challenge. So the Technology used to overcome this problem is Li-Fi (Light-Fidelity). The term Li-Fi refers to visible light communication (VLC) technology that uses light as a medium to deliver high-speed communication. Since visible light is present everywhere, the main idea is to create automatic indoor navigation systems for the visually impaired people using Li-Fi Technology.
\end{abstract}

Keywords: Li-Fi; VLC technology; high-speed communication; indoor navigation system.

\section{INTRODUCTION}

The light reaches everywhere and if certain information is to be passed using light as a medium, not only will the communication get fast but also the possibilities coming with it. Such a technique of using Light as a medium is named as the Li-Fi.

The Li-Fi technology can transfer the data through LEDs. It is a high speed and low cost wireless communication system, compared to $\mathrm{Wi}-\mathrm{Fi}$. It can provide high security, large bandwidth, and low cost. Li-Fi uses common household LED (light emitting diodes) light bulbs to enable data transfer, boasting speeds of up to 224 gigabits per second [1]. The concept of Li-Fi is currently attracting a great deal of interest, not least because it may offer a genuine and very efficient alternative to radio based wireless.

As a growing number of people and their many devices access wireless internet, the airwaves are becoming increasingly clogged, making it more and more difficult to get a reliable, high-speed signal. This may solve issues such as the shortage of radio-frequency bandwidth and allow internet where traditional radio based wireless isn't allowed such as aircraft or hospitals. Best part of visible light communication is that we can get advantage of high speed data communication at the same time we can use it for lighting purpose. In LI-FI light is used as a career as advancementto electromagnetic radio waves used in WI-FI. However it cannot penetrate walls, which the radio waves are able to. Li-Fi can be the technology for the future where data for laptops, smart phones, and tablets will be transmitted through the light in a room.

\section{PROBLEM STATEMENT}

People with visual disabilities, either partially or totally blind, are often challenged by places that are not designed for their special condition. Examples of these are bus and train terminals, hospitals and shopping malls. Several "everyday" objects that are present in most built environments become real obstacles for blind people, even putting at risk their physical integrity. Simple objects such as chairs, tables and stairs, hinder their movements and can often cause serious accidents. This Li-Fi technology helps the visually impaired to move within indoor environments. The main objective of the system is to provide, in real time, useful navigation information that enables a user to make appropriate and timely decisions on which route to follow in an indoor space.

Indoor navigation is convenient to everyone and it is especially indispensable for the visually impaired. $\mathrm{Li}-\mathrm{Fi}$ makes use of a free, unlicensed spectrum and is not affected by RF noise. Moreover, most indoor locations would have a sufficient amount of light sources and provide additional security since Li-Fi cannot penetrate through walls. The issues of the shortage of radio frequency bandwidth may be sorted out by Li-Fi and high data transmission rates of up to $10 \mathrm{Gbps}$ can be achieved.

With the increasing use of $\mathrm{Wi}-\mathrm{Fi}$, the existing radio frequency is getting blocked slowly and simultaneously, there are an increasing number of people who want to connect to the internet. LI-FI uses the Visible Light Communication (VLC). Visible light communication is a data communications medium which uses visible light and which will assist the visually impaired people for the navigation and helps them to travel independently at indoor places.

\section{III.OBJECTIVES}

The objective of the work is to create internal navigation systems for the bigger areas and also enables one to 


\author{
National Conference on Advances in Electrical Engineering \\ NMAM Institute of Technology, Nitte \\ Vol. 5, Special Issue 2, April 2017
}

identify the position of a person and calculates direction of his movements and it uses LED instead of bulbs and hence is indirectly helping environment to create automatic navigation for the visitors who are visually impaired.

\section{IV.SYSTEM DESIGN}

\section{A. Requirement Analysis}

The requirement analysis process involves deriving the system requirements through the observation of the existing system. A user interacts with the software through the user interface. The implementation of the software is of least importance to the end user [2]. Thus, any software designed should have a good user-interface maintaining the Integrity of the Specifications.

\section{B. System Configuration}

The wheelchair is intended to provide immense help to the person who is demonstrating as well as to the users of it. It therefore requires two configurations which are given below

Software Configuration:

- Windows /Linux Operating System

- $\quad$ Arduino IDE 1.5.7

Hardware Configuration:

- Arduino UNO ATMEGA 328P - It is a type of microcontroller that helps to build digital devices and interactive objects that can sense and control objects in the physical world.

- Voice play back circuit- Voice Module $60 \mathrm{Sec}$ project will playback and record up to 60 seconds of voice message. This circuit is an excellent choice to add voice to your project. Up to 60 Seconds of non-volatile voice storage.

- Ultrasonic Sensors- Ultrasonic ranging module HCSR04 provides $2 \mathrm{~cm}-400 \mathrm{~cm}$ non-contact measurement function , the ranging accuracy can reach to $3 \mathrm{~mm}$. The modules includes ultrasonic transmitter, receiver and control circuit.

- Transistors- A transistor is a semiconductor device used to amplify or switchelectronic signals and electrical power. It is composed of semiconductor material usually with at least three terminals for connection to an external circuit.

- LED Lamp-An LED lamp is a light-emitting diode (LED) product which is assembled into a lamp for use in lighting fixtures. A good LED driver can guarantee a long life for an LED system and provide additional features such as dimming and control.

L14F1 Photodiode-A photodiode is a semiconductor device that converts light into current. Photodiodes may contain optical filters, built-in lenses, and may have large or small surface

\section{METHODOLOGY}

Li-Fi Technology works by sending the data over light. For this purpose a LED (Light Emitting Diode) light bulb, anyone at all, can be flicked on and off in order to be able to generate signals. A proper Light Receiver is made for receiving the LED signals. The LED bulb will hold a micro-chip that will do the job of processing the data. The light intensity can be manipulated to send data by tiny changes in amplitude. The technology is focusing on making sure that the light bulb is flickered up to billions of times a second. At that time human eye cannot notice the light being flickered on and off. If the LED is ON, user can transmit a digital string of 1 , if it's OFF then user can transmit a string of 0 . It can be switched $\mathrm{ON}$ and $\mathrm{OFF}$ very quickly, which gives instant opportunity for transmitting data.

As shown in Fig.1, data is fed into LED light bulbs, it then sends data at rapid speeds to photodiode. The tiny changes in rapid dimming of LED bulbs are then converted by the receiver into electrical signal. The signal is then converted back into a binary stream that we could recognize as audio and video applications. High brightness LED which act as a communication source. The transmitter unit is fitted to the wall/ceiling. In the transmitter the modulated information is transmitted through LED.

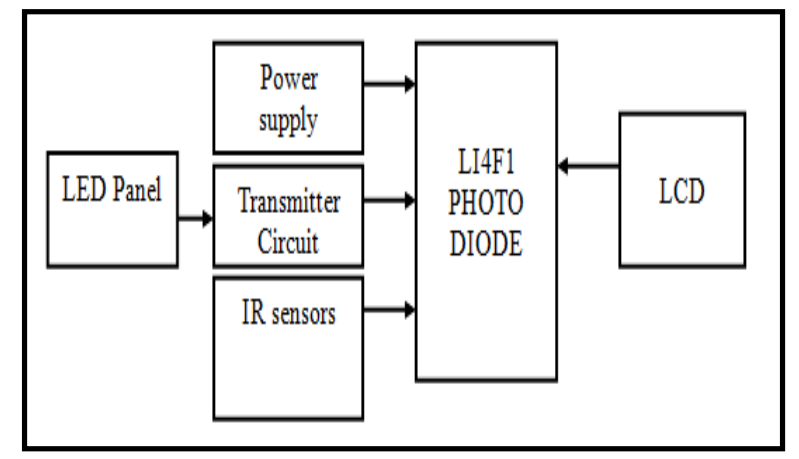

Fig.1Block diagram for Li-Fi data transmitter

As shown in the Fig. 2, thereceiver unit demodulates the encoded binary data and gives the response in the form of voice to the person [3]. The receiver unit consist of a photo transistor which receives the information from the LED connected to the transmitter. The information consist of location, and whenever receiver module comes in the range of that transmitter area than corresponding location message is send to receiver is stored in the IC ATMEGA328P and processed further to initiate voice to guide the person and vibrator motor to navigate the person which can assist visually impaired people at indoor places [4]. Transmitter Module - generates the corresponding onoff pattern for the LEDs. Receiver Module - has a photo diode to detect the on and off states of the LEDs. It captures this sequence and generates the binary sequence of the received signal. 


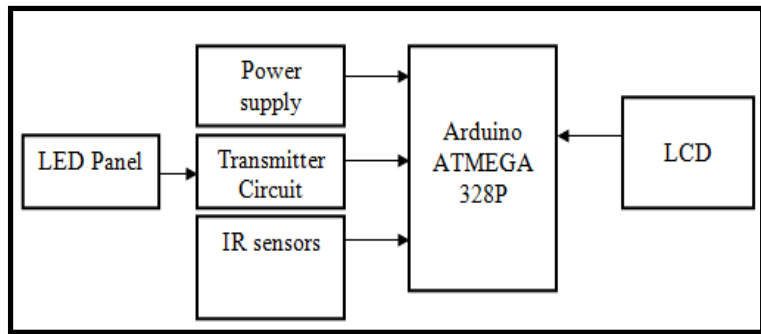

Fig.1Block diagram for Li-Fi data receiver

\section{VI.FUNCTIONAL DESIGN DOCUMENT}

TABLE I HARDWARE MODULE

\begin{tabular}{|l|l|}
\hline COMPONENTS & $\begin{array}{l}\text { Arduino ATMEGA } \\
\text { 328P,Voiceplayback,uv } \\
\text { sensor, led lamp }\end{array}$ \\
\hline PURPOSE & $\begin{array}{l}\text { Sense the person and helps } \\
\text { in indoor navigation }\end{array}$ \\
\hline ACKNOWLEDMENT & $\begin{array}{l}\text { Message to blind using } \\
\text { lifi(LED LAMP) }\end{array}$ \\
\hline
\end{tabular}

The Table-I will let us know about the initiation of the device, when the blind person enters into the room; transmitter device status is that the LED should be "ON". If it is not, then LED should be "OFF". Sensingthe environment Ultrasonic sensor detects the obstacles inside the room and Voice playback circuit speaks out the current location of the person. Action of the device is that If the LED is "ON" then transmitter circuit will send the data to the photodiode The tiny changes in rapid dimming of LED bulbs is then converted by the receiver into electrical signal [5]. The signal is then converted back into a binary stream that we could recognize as audio.

TABLE II SOFTwARE MOdule TRANSMitTER Circuit

\begin{tabular}{|l|l|}
\hline PARAMETERS & Add1,t,i \\
\hline RETURN & $\begin{array}{l}\text { Current location value } \\
\text { and obstacle detection }\end{array}$ \\
\hline VALUES & $\begin{array}{l}\text { To send the information } \\
\text { to blind person }\end{array}$ \\
\hline PURPOSE
\end{tabular}

TABLE III SOFTwARE MOdULE RECEIVER CirCuIT

\begin{tabular}{|l|l|}
\hline PARAMETERS & $\mathrm{BB}, \mathrm{x}, \mathrm{y}$ \\
\hline RETURN VALUES & $\begin{array}{l}\text { Captures on and off } \\
\text { state of led }\end{array}$ \\
\hline PURPOSE & $\begin{array}{l}\text { To send signal to } \\
\text { transmitter circuit for } \\
\text { the entry of the person }\end{array}$ \\
\hline
\end{tabular}

The Table-III will tell us the working of the software module and gives the brief description about it like what are the parameters used in program and also gives details about what are the return values and the main purpose of it

\section{RESULT AND CONCLUSION}

In addition to the uses of $\mathrm{Li}-\mathrm{Fi}$, it also satisfies the requirements of providing indoor navigation and also helps the visually impaired people to avoid obstacles and will also let them know about their current location. This technology will not only allow a user with visual disabilities to ambulate into an indoor environment while avoiding obstacles, but it could also help them interact with the environment. In the future we will not only have 14 billion light bulbs, we may have 14 billion $\mathrm{Li}$-Fi's deployed worldwide for a cleaner, greener and even a brighter future.

\section{REFERENCES}

[1] Jyoti Rani, PrernaChauhan, RitikaTripathi. "LiFi (Light Fidelity)The future technology in wireless communication.", International Journal of Applied Engineering Research, ISSN 0973-4562, 2012..

[2] Shubham Chatterjee, Shalabh Agarwal, AsokeNath, "Scope and Challenges in Light Fidelity(LiFi)Technology inWireless Data Communication.", International Journal of Innovative Research in Advanced Engineering,Issue 6,Vol 2.Richard Gilliard, Luxim Corporation, "The lifi lamp high efficiency high brightness light emitting plasma with long life and excellent colorquality".

[3] Richard P. Gilliard, Marc DeVincentis, AbdeslamHafidi, Daniel O'Hare,and Gregg Hollingsworth, "Operation of the LiFi Light Emitting Plasma".

[4] Visilink, "Visible Light Communication Technology for Near-Ubiquitous Networking”, White Paper, January 2012.

[5] J. Padhye, V. Firoiu, and D. Towsley, "A stochastic model of TCP Reno congestion avoidance and control," Univ. of Massachusetts, Amherst, MA, CMPSCI Tech. Rep. 99-02, 1999.

[6] Wireless LAN Medium Access Control (MAC) and Physical Layer (PHY) Specification, IEEE Std. 802.11, 1997. 